\title{
PELOTIZAÇÃO DO PÓ DE ACIARIA ELÉTRICA PARA OBTENÇÃO DE CONCENTRADO DE ZINCO EM FORNO WAELZ
}

\author{
Larissa Virgínia Queiroz Fagundes ' \\ Evandro Moraes da Gama ' \\ Luiz Henrique de Oliveira Martins ${ }^{2}$ \\ Roberto Galéry ' \\ Fabiana Maria Teixeira ${ }^{3}$ \\ Éder Lucio de Castro Martins ${ }^{3}$
}

\section{Resumo}

O presente trabalho teve por objetivo o estudo da pelotização do pó de aciaria elétrica (PAE), resíduo obtido na produção de aço através do forno elétrico a arco, como carga para forno Waelz, para obtenção de concentrado de zinco. Para caracterização do PAE foram realizadas análises granulométricas, análise química e análise mineralógica. Os resultados das análises granulométricas mostram grande heterogeneidade granulométrica, devido ao alto grau higroscópico do material que, na presença de umidade, tende a aglomerar. As análises química e mineralógica mostraram predominância de minerais de ferro e zinco. O PAE foi submetido a aglomeração a frio em disco pelotizador. Para determinação da resistência mecânica das pelotas, foram realizados ensaios de compressão uniaxial e drop number. Os resultados de resistência foram promissores e acima dos padrões inicialmente estabelecidos. Testes em forno piloto Waelz foram realizados para medir o impacto do uso das pelotas no processo. Os resultados dos testes em forno piloto também foram animadores, apontando para uma diminuição de acreção sem impactar no rendimento médio do zinco.

Palavras-chave: Pó de aciaria elétrica (PAE); Pelotização; Forno Waelz.

\section{PELLETIZING OF ELECTRIC ARC FURNACE DUST FOR OBTAIN ZINC CONCENTRATE IN WAELZ OVEN}

\begin{abstract}
The objective of the present work was to study the pelletizing of electric arc furnace dust, the residue obtained in the production of steel through the electric arc furnace, as a load for Waelz furnace, to obtain zinc concentrate. To characterize the dust, granulometric analysis, chemical analysis and mineralogical analysis were performed. The results of the granulometric analysis show a great granulometric heterogeneity due to the high hygroscopic degree of the material that, in the presence of moisture, tends to agglomerate. The chemical and mineralogical analyzes showed predominance of iron and zinc minerals. The PAE was subjected to cold agglomeration in disc pelletizing. To determine the mechanical strength of the pellets, uniaxial compression and drop number tests were performed. The resistance results were promising and above the standards initially established. Waelz pilot kiln tests were performed to measure the impact of pellet use in the process. The results of the pilot kiln tests were also encouraging, pointing to a decrease in accretion without impacting the average yield of zinc.
\end{abstract}

Keywords: Electric arc furnace dust; Pelletizing; Waelz Oven.

\section{INTRODUÇÃO}

Segundo a Associação Mundial do Aço [I], o Brasil é $\circ 9^{\circ}$ produtor mundial de aço. Atualmente, as indústrias de Aciaria Elétrica têm sustentado sua participação entre 20 e
25\% da produção nacional de aço [2]. Na siderurgia, assim como nas mais diversas atividades industriais, há uma intensa geração de resíduos. O pó de aciaria elétrica (PAE) é um

\footnotetext{
'Programa de Pós-graduação em Engenharia Metalúrgica, Materiais e de Minas, Universidade Federal de Minas Gerais. E-mail: larissaqueirozfagundes@gmail.com ${ }^{2}$ Programa de Pós-graduação em Engenharia e Gestão de Processos e Sistemas, Instituto de Educação Tecnológica - IETEC, Belo Horizonte, MG, Brasil ${ }^{3}$ Nexa Resources, Juiz de Fora, MG, Brasil
} 
resíduo particulado gerado em fornos elétricos. De acordo com Teo et al. [3], o PAE corresponde a I,5\% de toda carga enfornada em fornos de aciaria elétrica. A NBR I0.004 [4] classifica este resíduo como perigoso.

Da mesma forma que a legislação ambiental se torna mais rígida, cresce a necessidade de diminuição de custos e geração de receitas. As indústrias encontram inúmeras dificuldades para destinar corretamente seus resíduos em aterros. Diante disso, a necessidade do reaproveitamento de resíduos industriais é cada vez mais evidente na sociedade.

Segundo Zhiwei et al. [5], a constituição do PAE varia de acordo com a matéria-prima utilizada (sucata), mas pode ser constituída por óxidos de ferro, óxidos de zinco e sílica. A presença do zinco se deve a predominância de sucata de materiais galvanizados, como sucata de automóveis. Uma alternativa para reutilização desse material é a aplicação no processo produtivo de zinco.

A Votorantim Metais [6], na sua planta em Juiz de Fora/MG, volatiza o zinco presente no PAE em fornos rotativos através do processo Waelz. Trata-se de um processo pirometalúrgico para minérios de zinco e resíduos contento zinco, utilizado principalmente em resíduos da lixiviação neutra, PAE, escória da metalurgia de chumbo e resíduos silicatados de chumbo.

O teor de zinco existente no PAE, matéria-prima utilizada na Vototantim Metais [7], varia entre 17\% e 22\%. Após processo de volatização, o teor de zinco presente no óxido Waelz, produto do processo Waelz, fica acima de $55 \%$, enquanto que o teor de ferro deve ser menor que $4 \%$, gerando assim um concentrado de zinco adequado aos processos de concentração hidrometalúrgicos.

Durante processo Waelz alguns parâmetros são observados, na tentativa de garantir a qualidade do processo, são eles:

- Grau de enchimento do forno;

- Temperatura da escória;

- Acidez/basicidade da escória;

- Geração de carry over;

- Formação de acreção;

- Formação de big balls.

Uma das dificuldades do processo de volatização do zinco é a formação de acreção metálica nos fornos rotativos. As acreções dificultam a passagem natural do material dentro do forno. A formação das big balls é um problema para operação dos fornos Waelz, uma vez que aumenta o número de paradas para manutenção, aumentando os custos de operação e diminuindo a disponibilidade física dos fornos.

Atualmente na planta de Juiz de Fora/MG o processo de aglomeração do PAE é feito de forma concomitante ao processo de mistura das matérias-primas, em misturador vertical de alta rotação. Os aglomerados gerados, não apresentam grande resistência mecânica, gerando muitos finos durante etapas de carregamento e transporte. Acredita-se que a grande quantidade de finos presente no PAE facilite a formação de acreções nos fornos rotativos [6]. Na tentativa de minimizar a acreção foram aglomerados finos provenientes do PAE. Os ganhos no processo a partir da aglomeração a frio, com e sem adição de ligantes, são descritos no presente trabalho.

\section{MATERIAIS E MÉTODOS}

Para desenvolvimento do presente estudo sobre a aglomeração a frio de PAE em processo de volatização do zinco, foram desenvolvidas as seguintes etapas:

- Caracterização do PAE disponibilizado pela Votorantim Metais;

- Confecção de mistura a base de PAE, moinha de coque e ligante;

- Aglomeração a frio em disco pelotizador;

- Ensaios de resistência no material aglomerado/pelotizado;

- Adição dos aglomerados em processo de volatização do zinco em forno Waelz piloto;

- Ganhos obtidos no processo de volatização do zinco.

\section{I Caracterização do PAE}

A caracterização física e química do PAE foi realizada por técnicas de caracterização mineralógica tecnológica, são elas: análise granulométrica, análise química por fluorescência de raios $X$, análise mineralógica por difratometria de raios $X e$ análise granulométrica microscópica por cyclosizer.

Todas estas técnicas foram realizadas nos laboratórios do Departamento de Engenharia de Minas da Universidade Federal de Minas Gerais.

\subsection{Confecção de Misturas}

Após caracterização físico-química do PAE, foi possível determinação de ligante inorgânico ideal para mistura. Para tanto, foram confeccionadas misturas a base de PAE e moinha de coque. $O$ equipamento utilizado para mistura em escala de bancada foi o misturador WAM 30 litros horizontal. Já para mistura em escala piloto, utilizou-se o misturador WAM 505 litros horizontal.

Para melhorar a performance de resistência mecânica das pelotas, foram realizados ensaios de aglomeração com e sem a adição de ligante inorgânico a base de estéreis de mineração de ferro.

\subsection{Aglomeração a Frio}

Após o processo de mistura foram confeccionados ensaios de aglomeração em disco pelotizador. Os ensaios em escala de bancada foram realizados em disco pelotizador de $40 \mathrm{~cm}$ 
de diâmetro [7]. Para os ensaios em escala piloto foi utilizado disco pelotizador Haver Scarabeus ${ }^{\circledR}$, com I m de diâmetro, borda regulável, totalmente automatizado. $O$ processo de cura foi feito em ambiente. Para acompanhamento da evolução da resistência mecânica do aglomerado durante etapa de cura, foram realizados ensaios de resistência à compressão uniaxial com 0,24 e 48 horas de cura.

\subsection{Ensaios de Resistência Mecânica}

O acompanhamento da resistência mecânica foi feito durante o processo de cura, nos tempos 0, 24 e 48 horas. A faixa granulométrica utilizada foi entre $9,5 \mathrm{~mm}$ e $12,5 \mathrm{~mm}$. A população testada foi de 15 unidades para cada ensaio.

\subsection{Resistência à compressão uniaxial}

Para determinação da resistência à compressão uniaxial foi utilizada prensa controlada. A prensa comprime a pelota até que haja rompimento ou fissura aparente.

\subsubsection{Resistência à resiliência}

Para indicação da resistência à resiliência foi utilizado ensaio de drop number, com haste metálica de $40 \mathrm{~cm}$ de comprimento. A pelota é derrubada da parte superior da haste em superfície metálica até que seja observada fissura aparente.

\subsection{Adição de Aglomerados em Forno Waelz Piloto}

Após período de cura de no mínimo 48 horas, os aglomerados foram adicionados em forno Waelz piloto. A Tabela I traz um comparativo entre o forno piloto e o forno industrial utilizados na Votorantim Metais em Juiz de Fora.

As reações principais ocorrem na zona de reações principais, em um intervalo de temperatura que vai de $900^{\circ}$ a $1200^{\circ} \mathrm{C}$. Foram observados parâmetros como rendimento do forno e formação de acreção.

\section{RESULTADOS E DISCUSSÃO}

Os resultados deste trabalho são descritos a seguir.

\section{I Resultado da caracterização do PAE}

A difração de raios $X$ e a fluorescência de raios $X$ foram feitas no Laboratório de Raios $X$ do Departamento de Engenharia de Minas da UFMG. A difração de raios $X$ identificou, principalmente, a existência de franklinita ((Zn,Mn,Fe)204)), zincita $((\mathrm{Zn}, \mathrm{Mn}) 0)$ e silvita $(\mathrm{KCl})$, conforme Figura I.

Conforme previsto, através da técnica de fluorescência de raios $X$, foram encontrados principalmente $\mathrm{Fe}(36,1 \%)$, $\mathrm{Zn}(15,2 \%), \mathrm{Ca}(9,7 \%)$, além de traços de $\mathrm{Pb}$ e $\mathrm{Cd}$. A grande quantidade de ferro e zinco no PAE se dá graças ao predominante uso de sucata galvanizada como matéria-prima para os fornos a Arco Elétrico em estudo.

A análise granulométrica pela série normal de Tyler mostrou que há grande dispersão granulométrica entre as partículas. Isso ocorre graças a umidade presente na amostra, facilitando assim a aglomeração do material. Aproximadamente $47 \%$ do material encontra-se em frações superiores a I, $2 \mathrm{~mm}$, enquanto que cerca de $16 \%$ encontra-se na fração fina, abaixo de $75 \mu \mathrm{m}$, conforme Figura 2.

Com isso, tem-se a necessidade de implantação de uma etapa de peneiramento para garantir assim, a uniformidade do material a ser pelotizado.

Tabela I. Comparativo entre forno industrial e forno piloto [7]

\begin{tabular}{lcc}
\hline \multicolumn{3}{c}{ PARALELO FORNO INDUSTRIAL X FORNO PILOTO } \\
\hline & Forno Industrial & Forno Piloto \\
\hline Diâmetro (m) & 4 & $0,3 \mathrm{I}$ \\
Comprimento (m) & 70 & 4 \\
Inclinação $\left(^{\circ}\right)$ & 2 & 2 \\
Rotação (rpm) & 0,4 a I,I & 0,4 a I, I \\
Tempo de residência (h) & 8 & 8 \\
\hline
\end{tabular}

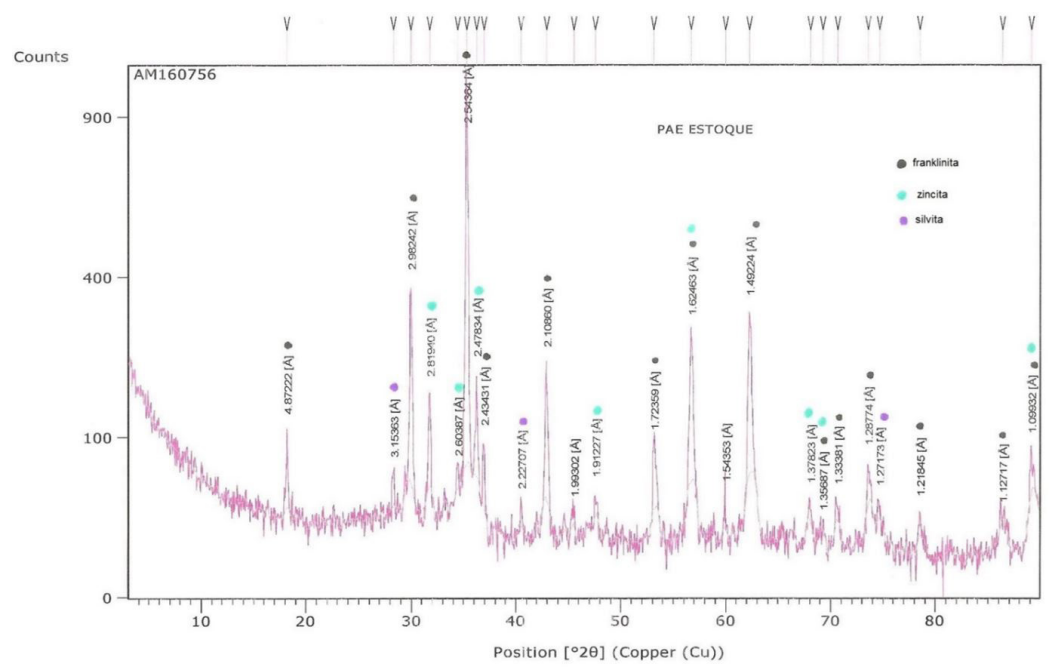

Figura I. Difratometria de raios $X$ amostra PAE [8]. 
A análise granulométrica feita pelo cyclosizer caracterizou a fração superfina da amostra, conforme Figura 3.

Para determinação da granulometria do ligante a ser empregado, o cyclosizer teve grande importância, caracterizando a fração superfina da amostra. Cerca de $50 \%$ da amostra se encontra na fração superfina abaixo de 30 micrômetros.

\subsection{Resultado da Confecção das Misturas}

Foram confeccionadas duas misturas. A mistura I composta por $95 \%$ de PAE e $5 \%$ de moinha de carvão e a mistura II, composta por $89 \%$ de PAE, $5 \%$ de moinha de carvão e $6 \%$ de Metkaflex, ligante inorgânico, ainda em fase de pesquisa e desenvolvimento pelo Laboratório de Geotecnologia e Geomateriais da UFMG, proveniente da calcinação de estéreis da mineração de ferro. A Tabela 2 contém os parâmetros utilizados no processo de mistura. A mistura foi feita em misturador piloto WAM horizontal de 505L.

A base da mistura I (PAE + moinha de carvão) foi sugerida pela Nexa Resourses, na tentativa de reproduzir os parâmetros aplicados na planta industrial. Já a mistura II, foi confeccionada com a quantidade limite de aglomerante suportada pelo processo, sem que houvessem perdas de produtividade.

\subsection{Resultado da Aglomeração a Frio}

Após processo de mistura o material foi pelotizado em disco pelotizador piloto Haver, modelo Scarabeus ${ }^{\circledR}$. Foram observados parâmetros de inclinação, rotação, nível de vibração da calha, vazão de água, tempo de ensaio, entre outros. $\mathrm{Na}$ Tabela 3 são mostrados alguns parâmetros adotados.

O tamanho de pelotas considerado ótimo foi entre $12,5 \mathrm{~mm}$ e $9 \mathrm{~mm}$, granulometria adotada para pelotas de minério de ferro. Todavia, para um estudo mais aprofundado da relação volume da pelota/ melhoria no processo, foram geradas pelotas em várias faixas granulométricas. A Figura 4 mostra pelotas geradas a partir da pelotização do PAE.

É possível que a presença de aglomerante na mistura, seja responsável pelo breve aumento da umidade de pelotização, II\% na mistura I (sem aglomerante) e I I,5\% na mistura II (com aglomerante).

\subsection{Resultado dos Ensaios de Resistência Mecânica}

Para avaliação da resistência das pelotas quando dispostas em pilhas, foi realizado teste de resistência à compressão uniaxial. Já para avaliação da resistência das pelotas aos tombos, durante transporte, foi realizado teste de resiliência.

\subsection{Resultado do teste de resistência à compressão}

O resultado do teste de resistência à compressão é mostrado na Tabela 4.

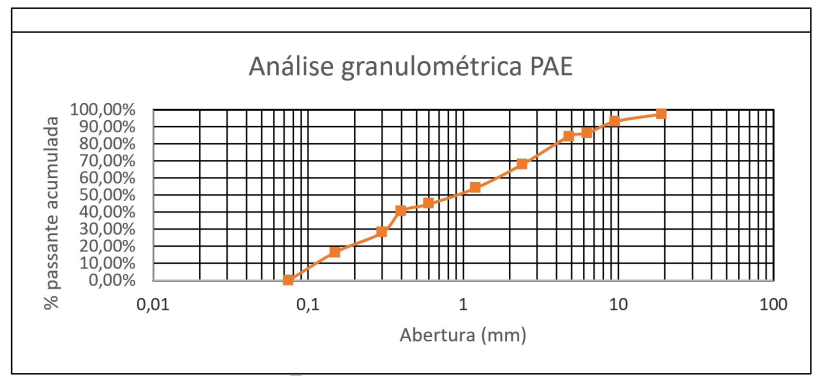

Figura 2. Análise granulométrica PAE [8].

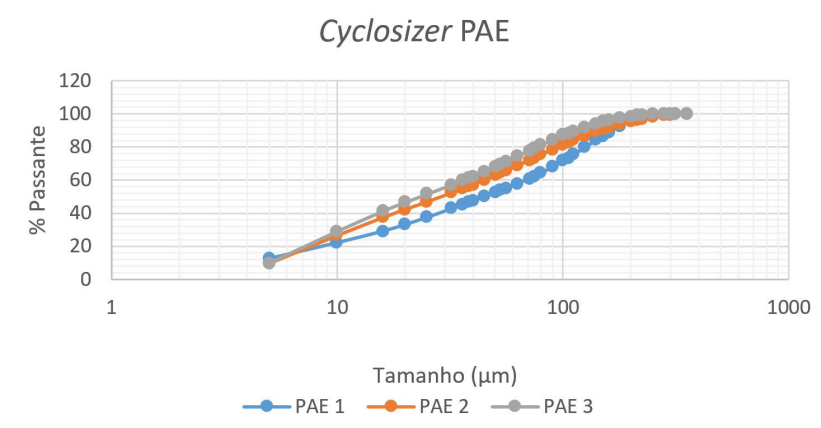

Figura 3. Análise granulométrica em Cyclosizer [8].

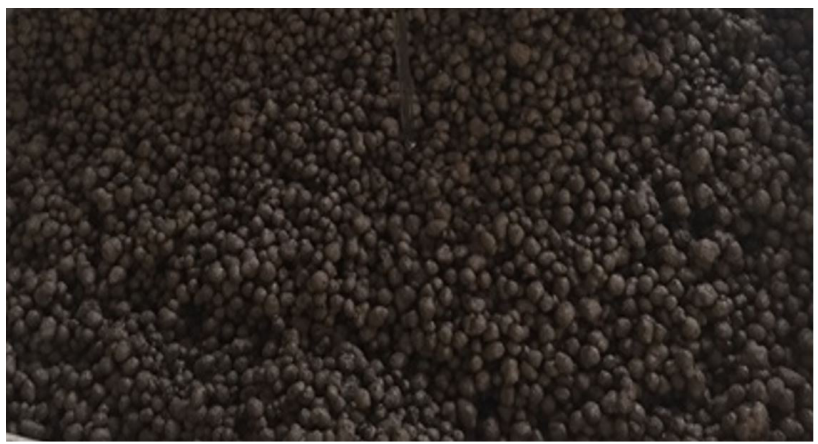

Figura 4. PAE pelotizado [8].

Tabela 2. Parâmetros do processo de mistura [8]

\begin{tabular}{lcccc}
\hline Amostra & $\begin{array}{c}\text { Rotação } \\
(\mathbf{r p m})\end{array}$ & $\begin{array}{c}\text { Tempo de } \\
\text { mistura } \\
(\mathbf{m i n})\end{array}$ & $\begin{array}{c}\text { Umidade } \\
\mathbf{( \% )}\end{array}$ & $\begin{array}{c}\text { Grau de } \\
\text { enchimento } \\
(\%)\end{array}$ \\
\hline Mistura I & 200 & 10 & $9 \%$ & $50 \%$ \\
Mistura II & 200 & 10 & $9,3 \%$ & $50 \%$ \\
\hline
\end{tabular}

Tabela 3. Parâmetros do processo de pelotização [9]

\begin{tabular}{lcccc}
\hline \multicolumn{5}{c}{ Pelotização } \\
\hline Amostra & $\begin{array}{c}\text { Rotação } \\
\text { (rpm) }\end{array}$ & $\begin{array}{c}\text { Tempo } \\
\text { de ensaio } \\
\text { (min) }\end{array}$ & $\begin{array}{c}\text { Umidade } \\
\text { (\%) }\end{array}$ & $\begin{array}{c}\text { Inclinação } \\
\text { ( })\end{array}$ \\
\hline Mistura I & 18 & 74 & $11 \%$ & 54 \\
Mistura II & 18 & 68 & $11,5 \%$ & 54 \\
\hline
\end{tabular}


Tabela 4. Teste de resistência à compressão uniaxial [8]

\begin{tabular}{ccc}
\hline \multicolumn{2}{c}{ Teste de resistência (kgf/pelota) } & $-\mathbf{2 , 5} \mathbf{m m}+\mathbf{9 , 5 m m}$ \\
\hline $\begin{array}{c}\text { Amostra com } \mathbf{4 8} \\
\text { horas de cura }\end{array}$ & Média & Desvio padrão \\
\hline Mistura I & $\mathrm{I}, 67$ & 0,36 \\
Mistura II & 2,13 & 0,38 \\
\hline
\end{tabular}

Tabela 5. Teste de resistência à resiliência [8]

\begin{tabular}{ccc}
\hline \multicolumn{2}{c}{ Teste de resiliência (n ${ }^{\circ}$ quedas/pelota) } & $-\mathbf{I 2 , 5} \mathbf{m m}+\mathbf{9 , 5 m m}$ \\
\hline $\begin{array}{c}\text { Amostra com } \mathbf{4 8} \\
\text { horas de cura }\end{array}$ & Média & Desvio padrão \\
\hline Mistura I & 7 & 0,87 \\
Mistura II & 5 & 0,9 \\
\hline
\end{tabular}

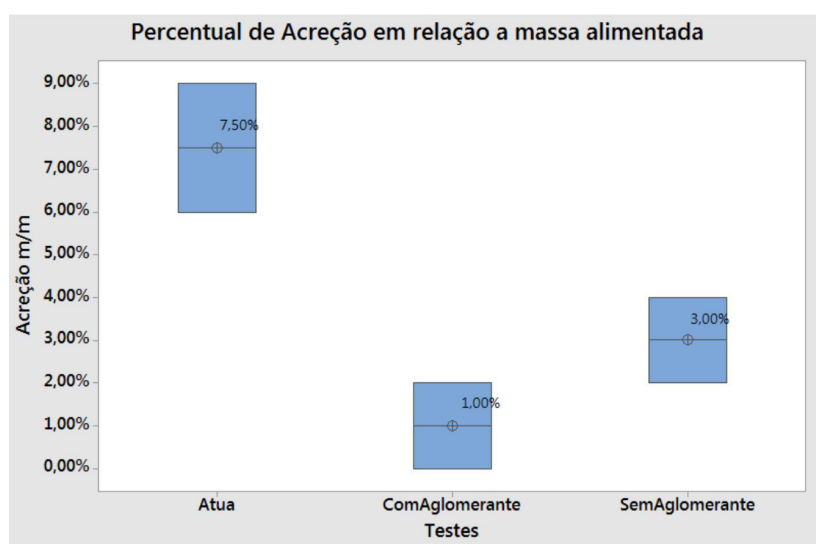

Figura 5. Percentual de acreção gerado [6].

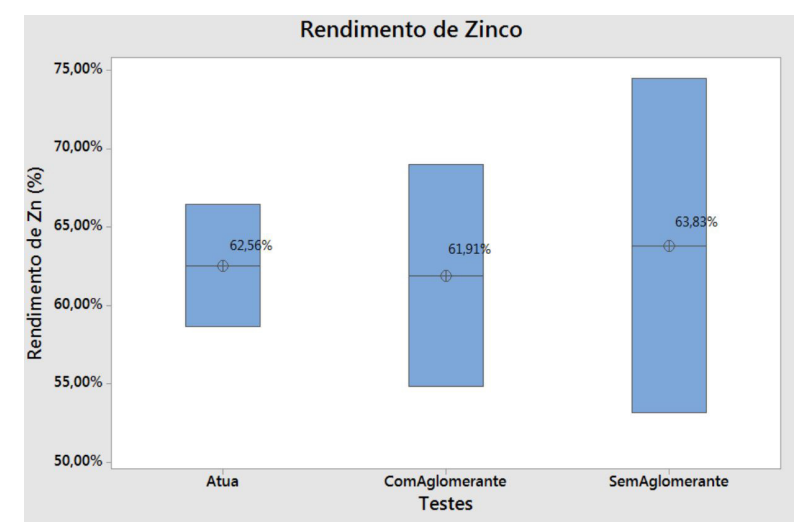

Figura 6. Rendimento de zinco [6].

Os valores de resistência obtidos após 48 horas de cura foram superiores aos parâmetros estabelecidos pela Votorantim Metais, entre 0,8 kgf e I,2 kgf. A adição do ligante elevou em quase $30 \%$ a resistência à compressão das pelotas.

\subsubsection{Resultado do teste de resistência à resiliência}

O resultado do teste de resistência à resiliência é expresso na Tabela 5.

Em ambos os testes os valores foram considerados bons e acima dos praticados na unidade Juiz de Fora.

\subsection{Resultado da adição de aglomerados em forno Waelz piloto}

Foram testadas 3 situações de operação, são elas:

I -Operação sem pelotas (atual);

2 - Operação com pelotas da mistura I (sem aglomerante);

3 - Operação com pelotas da mistura II (com aglomerante).

Os resultados obtidos são descritos nas Figuras 5 e 6.

$A$ adição de aglomerante diminui o percentual de acreção gerada, de 7,5\% (situação atual) para $1 \%$ (testes com uso de aglomerante), todavia este não conseguiu elevar o rendimento de zinco no processo. Já os testes sem o uso de aglomerante indicaram uma boa queda na geração de acreção de $7,5 \%$ (situação atual) para $3 \%$, somado ao bom rendimento de zinco, de $62,6 \%$ (situação atual), para $63,8 \%$.

Segundo parâmetros operacionais adotados na usina, o rendimento médio de zinco deve ser superior a $60 \%$. Em todos os testes o mínimo aceitável foi obedecido.

\section{CONCLUSÃO}

Como conclusão deste trabalho têm-se:

- Para as condições ensaiadas em escala de bancada, os testes se mostraram animadores, denotando grande potencial para aplicação em escala industrial;

- A aglomeração a frio do PAE em disco pelotizador mostrou-se viável e tecnicamente a melhor opção, uma vez que se tem uma maior uniformidade física das pelotas, possibilitando assim maior controle nos processos produtivos;

- As pelotas geradas apresentaram, entre outras características físicas, resistência mecânica compatível à metalurgia do zinco;

- O uso do Metkaflex incentiva a mineração circular, uma vez que se trata de estéril gerado pela mineração de ferro;

- A geração média de acreção em testes onde foram utilizadas pelotas sem adição de ligantes foi 2 vezes menor que os testes onde não foram utilizadas pelotas. Já os testes de pelotas com ligante, a acreção gerada chegou a ser 7 vezes menor se comparada aos testes sem adição de pelotas;

- A alimentação do forno com pelotas não causou grande impacto no rendimento de zinco. Em todos os testes o rendimento esteve acima de $60 \%$, padrão estabelecido pela Votorantim Metais.

\section{Agradecimentos}

Os autores agradecem a UFMG ao apoio no desenvolvimento deste trabalho em conjunto com a Nexa Resources. 


\section{REFERÊNCIAS}

I Worldsteel Association [página da internet]. World steel in figures 2019 [acesso em 12 ago 2019]. Disponível em: https://www.worldsteel.org/en/dam/jcr:96d7a585-e6b2-4d63-b943-4cd9ab62 la9l/World\%2520Steel\%2520in\%25 20Figures\%25202019.pdf

2 Telles V, Junca E, Rodrigues GF, Espinosa DCR, Tenório JAS. Utilização da poeira de aciaria elétrica na sinterização de minério de ferro: influência da granulometria. In: Anais do $19^{\circ}$ Congresso Brasileiro de Engenharia e Ciência dos Materiais - CBECiMat; 2010 nov; Campos do Jordão, Brazil. São Paulo: editora. p. 7380-7387.

3 Teo YY, Lee HS, Low YC, Choong SW. Hydrometallurgical extraction of zinc and iron from electric arc furnace dust (EAFD) using hydrochloric acid. Journal of Physiological Sciences. 2018;29(3):49-54.

4 Associação Brasileira de Normas Técnicas. NBR 10004: Resíduos Sólidos - classificação. Rio de Janeiro: ABNT; 2004.

5 Zhiwei P, Lin X, Yan J, Hwang J-Y. Characterization of low - zinc electric arc furnace dust. In: Ikhmayies S, Bowen L, Carpenter JS, Li J, Hwang J-Y, Monteiro SN, et al. Characterization of minerals, metals and materials. USA: Springer; 2017. p. $103-109$.

6 Votorantim Metais. Adequação da qualidade das pelotas. Desenvolvimento tecnológico. Juiz de Fora: Votorantim Metais; 2017. 21 p.

7 Votorantim Metais. Resultados dos testes no forno Waelz. Desenvolvimento tecnológico. Juiz de Fora: Votorantim Metais; 20I7. II p.

8 Haver \& Boecker. Testes disco pelotizador Scarabeus. Pedro Leopoldo: Engenharia, Gerência de Engenharia Documentos internos; 2017. 25 p.

9 Laboratório de Geotecnologia e Geomateriais da UFMG. Relatório final. Belo Horizonte: Centro de Produção Sustentável; 20I7. 46 p.

Recebido em: 16 Jan. 2019

Aceito em: I5 Set. 2019 